\title{
The Subtle Organic Brain Inventory of Neppe (SOBIN): a necessary tool in the neuropsychiatric population and a valuable one in psychiatry and psychology: clinical and forensic applications
}

\section{Abstract}

The SOBIN (Subtle Organic Brain Inventory of Neppe) is the only available clinical neuropsychiatric inventory screening for soft organic type symptoms and learning disabilities. It was developed in 2002 by Vernon Neppe at the Pacific Neuropsychiatric Institute in Seattle, where it's used extensively.

The SOBIN is a paper-and-pencil screen of pertinent medical history relating to higher brain function. It is designed to integrate six major elements:

a. Primarily, the items elicit soft organic brain pathology. This is particularly important in the broad spectrum of neuropsychiatric cases ranging from possible brain injury, to psychopathology, to soft organic impairments that may have been lifelong, such as in evaluating subtle difficulties like prosopagnosia, dysproccia and attention deficits.

b. Secondly, the SOBIN allows for monitoring patient-reported changes, including after significant pathology like head injury or encephalitis

c. Thirdly, the SOBIN provides awareness of the subjective cognitive areas of special strengths.

d. Fourthly, the questions elicit monitoring ordinal severity fluctuations in higher brain function. This is particularly useful in medicolegal cases, as well as cases where distinct events such as traumatic brain injury occurs

e. Fifthly, the SOBIN assists with obtaining ancillary baseline data, ranging from laterality to personality to symptom triggers, additional to the INSET (Inventory of Neppe of Symptoms of Epilepsy and the Temporal Lobe).

f. Sixth, we have provisionally clustered the SOBIN items into 11 broad categories, modelling it on Vernon Neppe's BROCAS SCAN, an instrument that objectively clinically measures higher brain functions. By these means we can compare the subjective and objective results, though the items historically measure broader and sometimes different items than that of the BROCAS SCAN.

The SOBIN provides a consistent and standardized method of measuring reports of higher brain symptoms. It is most often combined with another of Vernon Neppe's screens, the INSET.

Together and along with the clinical examination, these tests assist the clinician in ensuring there are no indicators of invalid responses. This way one is able to better interpret neuropsychiatric tests properly. The SOBIN has unique neuropsychiatric use and applications because it is the only instrument in its class.1-3

By scoring 88 main items plus 24 ancillary items, the SOBIN-1 compares past and present, evaluates degrees of severity, and proves critical in both clinical and forensic contexts, including patients with questionable organic or brain pathology or neurological conditions with possible psychiatric elements, previous head injury or encephalitis or tumor or other brain insult or possible seizure disorders or paroxsymal neurobehavioral disorders. Further items were recently added, making up an extra 112 questions and called the SOBIN-2. The SOBIN is the name for the 200 -item combination of the SOBIN-1 and the SOBIN-2. Effectively the two SOBINs (1 and 2) are now used together as a single instrument, the SOBIN.

The SOBIN is the main focus here. However, because it has always been used with the INSET and with an objective evaluation of higher brain function, the BROCAS SCAN, and the 10 higher brain categories of the ROCAS applied to cluster analysis of symptoms, the INSET and BROCAS SCAN (along with the MOCA and MMSE) are included in some detail.

We also apply two neglected conditions-prosopagnosia and dysproccia — as examples of how to apply clinical data and ultimately research using the SOBIN.
Volume 9 Issue 3 - 2018

Vernon M Neppe MD, PhD, FRS(SAf), DFAPA DPM, MMed, DPCP (ECAO), DSPE, ${ }^{1,2}$ Shauna Mason $\mathrm{BS}^{3}$

'Director, Pacific Neuropsychiatric Institute and Exceptional Creative Achievement Organization, USA

2Department of Neurology and Psychiatry, St. Louis University, USA

${ }^{3}$ Senior Research Assistant, Pacific Neuropsychiatric Institute, USA

Correspondence: Vernon M Neppe, Director, Pacific Neuropsychiatric Institute and Exceptional Creative Achievement Organization, Seattle, Washington, USA, Tel 206527-6289,Email psyche@pni.org; www.pni.org

Received: April 16, 2018 | Published: May 21, 2018 


\section{Perspective}

The SOBIN (Subtle Organic Brain Inventory of Neppe) has become critically important because there remains no other detailed test to screen for subtle organic brain symptoms. It was developed out of necessity. We have used it at the Pacific Neuropsychiatric Institute in Seattle, WA since 2012, applying this historical symptom screen in almost every patient. ${ }^{1-3}$ We have found the SOBIN has enormous relevance while applying it to hundreds of patients.

It has become extremely important to evaluate differences in events, particularly if any specific brain injury is involved. The SOBIN appears sensitive to what it purports to measure (symptoms pertaining to higher brain function) and patients find the items easy to understand.

\section{Populations}

The SOBIN (Subtle Organic Brain Inventory of Neppe) has become critically important because there remains no other detailed test to screen for subtle organic brain symptoms. It was developed out of necessity. ${ }^{1-3}$ We have used it at the Pacific Neuropsychiatric Institute in Seattle, WA since 2012, applying this historical symptom screen in almost every adult patient. Our population has generally been between the age ranges of 16 and above through 65 and rarely into the eighties. These males or females have a broad range of diagnoses from intact higher brain functioning through to profoundly brain damaged with a broad spectrum of psychopathology and functioning. We have found the SOBIN has enormous relevance while applying it to hundreds of patients, always with other testing and detailed clinical evaluations.

It is often important to evaluate differences over time, particularly if any possible brain injuries have occurred. The SOBIN appears sensitive to what it purports to measure (symptoms pertaining to higher brain function), is reliable, and apparently sensitive to dysfunctions. Patients find the items comprehensible. It, therefore, contributes markedly to the evaluation.

\section{Importance}

The SOBIN is greatly important for both clinical and forensic patients in Neuropsychiatry and Behavioral Neurology. Apparently, almost everyone has some difficulties at a higher brain level, even ostensibly normal individuals. Effectively, we all are wired slightly differently, with weaknesses that are compensated for by strengths and sometimes strengths that allow our brains to perform in exceptional manners.

\section{Applications}

Because everyone, based on our data, has higher brain dysfunctions as well as likely compensating strengths, it's useful even in the Psychiatric patient. For those with specific or more general higher brain deficits, we can apply the SOBIN along with medical, psychological, counseling, and occupational therapy features to provide directions for cognitive rehabilitation. Therefore, the SOBIN is a versatile way to approach patients in Psychiatry, Neurology, Psychology and of course, it's primary population of Neuropsychiatric and Behavioral Neurological patients, both clinical and medicolegal.

\section{The SOBIN: special concepts}

\section{Clustering principles}

The symptoms elicited in the SOBIN can be clustered together, for example, to allow more detailed evaluations such as "dysproccia" (a disorder of processing afferent and efferent information), ${ }_{1}^{1}$ attentional disturbances, focal disturbances such as dyspraxia and dysgnosia, as well as prosopagnosia, right-left, directional and many other subtle disturbances. Particularly important are changes that occur for example, "normal" to "gross" after cerebral insults

\section{Exceptional scoring}

Also pertinent is utilizing any self-rated "exceptional" abilities. There is great value to knowing this, as such skills can be applied, in for example, rehabilitation. Patients scores are obviously different for each item, but the overall scores can be totaled giving a perspective on Gross impairment, Slight impairment, "Normal", and also Exceptional Skills.

\section{Measuring progression}

A new variant in the SOBIN, is obtaining an extra column of information, useful after possible brain trauma. The patient ranks how badly they were doing after the brain insult, but then ranks progression currently as worse, better, or the same.

\section{History of the SOBIN}

The SOBIN (Subtle Organic Brain Inventory of Neppe) is a unique inventory, because rather remarkably, no one else has apparently used a way to clinically evaluate by structured history taking, the multiple different facets of organic brain injury. Even those without direct and obvious impairments invariably have some subtle impairmentsto the extent that we have never, since the SOBIN began in 2002, encountered any patient who did not admit to previous slight or gross difficulties on at least one item. We have performed SOBINs routinely and therefore on hundreds of patients - new patientssometimes repeating it, but not necessarily so, and only unusually. This is because organic brain injuries, such as certain dysfunctions, do not tend to change dramatically over time. However, this is an area for future research. Very few patients have difficulty with this test, which they can do independently of even our assistants except for the occasional questions.

The derivation of this SOBIN instrument came about by the experience of Professor Vernon Neppe over many years, noting the difficulties his patients were having. Also, an extended literature review was performed, although most times many of the features of the literature review did not show up the kinds of symptoms that we were trying to screen.

The SOBIN-2 has only just been completed, in April of 2018. So we have little experience with it. But effectively, the SOBIN-2 is just an extension of the SOBIN and its value was recognized during the re-examination consequent on the writing of this paper.

\section{Validity}

The SOBIN therefore cannot be compared with other standard instruments for validity because comparisons don't exist. However, given our extensive 16-year experience with this paper-and-pencil instrument, we know the following: It has construct, content and criterion validity plus face validity and reliability

1. It has face validity. This refers to the degree to which this neuropsychological assessment appears effective in terms of its stated aims: The SOBIN certainly is effective in what it does. 
2. It has construct validity. This refers to the degree to which the SOBIN measures what it claims, or purports, to be measuring. It is valid in the context of reflecting what symptoms the person has, and when one clinically evaluates them, one finds that these symptoms are appropriate.

3. The SOBIN has content validity: This means it has logical validity to the extent to which the SOBIN measures all facets of the given construct of subtle higher brain impairments.

4. It has criterion validity in that there is a concrete that is related to the outcome of measuring higher brain impairments. This criterion validity is sometimes subdivided into concurrent validity where the clinical and research published evidence can defend the use of the SOBIN for predicting other outcomes (such as cerebral cortical organic impairments) and predictive validity where the informal cognitive test correlations between the SOBIN scores and the expert clinical neuropsychiatrist opinion appears very high.

5. It is reliable in that patients will respond to the same question approximately the same way each time: It is consistent. That repetition has been done though not required most times.

6. It has forensic uses, which are very important, particularly after an event, such as a form of traumatic brain injury or other forms of injury or systemic phenomena. The current SOBIN revision has three major columns:

a. Before/past, which can include childhood.

b. Immediately following the event, such as an injury.

c. The results thereafter, the current status.

7. It also allows us to evaluate certain dysfunctions. Among those dysfunctions that we briefly look at here are two that have been very much neglected. One is the component of not recognizing difficulties with processing information: Sometimes people use words like 'dyslexia' or 'acalculia' or 'ADHD' but in fact, much of this involves both executive and/or perceptual experiences; and sometimes the experiences are central within higher brain dysfunction.

\section{Measurements}

We are able to get an index in terms of severity - for example 'slight' and 'gross' - and we are also able to evaluate any 'exceptional' skills. Many patients will report gross impairments at times, sometimes subtle impairments, and recognize them and compensate; and some of them will report that they have certain exceptional skills. This is very important clinically and forensically because one can use the patient's strengths to work with change. We also can ask the patient to list their difficulties before and afterwards an event, such as head trauma. This has proved very, very valuable. Finally, from the patient's report of status after the injury, we elicit the current situation (unchanged, worse, better)

\section{Accessory features to the SOBIN}

Additionally, there is great value in assessing such accessory features as mixed laterality, responses to stimuli like lights, and the patient's self-evaluations like intelligence.

The SOBIN-1 contains two-dozen accessory questions and symptoms. These do not directly relate to higher brain functioning abnormalities; but they must be perceived in the context of the SOBIN being administered with another instrument, the 'INSET',which preceded the SOBIN by some 20 years. Consequently, some of the items in the INSET, despite a further major revision in between, but still preceding the SOBIN, are not in the INSET but are in the SOBIN instead, because the two screens are used adjunctly. In addition, we added 10 more items on predisposing causes A to J in the SOBIN-2, making up 34 accessory items. Effectively the two SOBINs (1 and 2) are now used together as a single instrument, the SOBIN. In these accessory items, we ask about the patient's strengths. We request them to do a self- assessment of their own intelligence-very useful to obtain one component of a perspective of the patient's self-awareness.

We ask about whether or not they feel they have responded to things honestly - again valuable, because if not, we might discount much of the history.

We also ask about handedness at every level of laterality, because we were interested in what proportion of our population had mixed laterality features and what that might reflect diagnostically (for example, is Attention Deficit Disorder more common?)

One of the questions that we were asking related to how one sleeps (on what side? prone? supine? On the right; on the left?). However, we have no data at this point that this has any linkup with laterality. It might however have a linkup with such features as sleep apnea. So, the SOBIN allows us to find out extra information that is valuable, which we can apply.

In retrospect, some aspects of the SOBIN are not best placed there but should have been in the INSET, but the INSET was already completed so the SOBIN had some logical INSET items. We look at triggers of episodic phenomena. Logically, these would have been best placed in the INSET, but the INSET has not been further revised: These include flashing lights that are potentially important triggers and we then ask about any kinds of sensory phenomena. We then want to elicit predisposing phenomena. For example, at the end of the SOBIN we ask about difficulty with flashing lights, and this synchronization is often an acute symptom relating to some kind of seizure phenomenon.

\section{Adjunctive tests with the SOBIN}

The SOBIN can be used as a routine instrument in every instance to evaluate neuropsychiatric and behavioral neurological conditions more correctly.

However, symptoms relating to the brain should seldom be perceived in isolation. Consequently, Neppe developed the SOBIN as a historical paper-and-pencil office waiting-room instrument. It was intended to be used alongside appropriate different instruments.

Three tests are commonly used together when the SOBIN is used.

a. Initially, when the SOBIN is given the INSET is almost always filled in, as well.

b. The BROCAS SCAN is an objective examination administered at least once. to almost every neuropsychiatric patient. We currently also perform items from the MOCA and Folstein MMSE so as to be able to compare results, although we're not finding either of the latter essential. 


\section{The INSET: its common application with the SOBIN}

The SOBIN is seldom performed on its own. It is an instrument that involves a cluster, and one important part of the cluster is the aforementioned INSET (the Inventory of Neppe of Symptoms of Epilepsy and the Temporal Lobe).

The INSET screening questionnaire was also developed out of necessity in the running of the Pacific Neuropsychiatric Institute. Vernon Neppe authored an early version of this test in 19772 and adapted it through to 19923 . It has become critically important because there remains no test to screen for possible temporal lobe symptoms. Subsequently, others have tried to adapt this but we have used it at the Pacific Neuropsychiatric Institute in Seattle, WA as a screen in almost every patient. The availability of being able to use this as a jumping point for more detailed clinical history taking is very relevant. Dr. Neppe lists below the INSET, which is actually the SHORT INSET. (The Long INSET4 also exists but is seldom used because the INSET requires some expertise for its useful clinical application3.) It has become greatly important with ambulatory encephalography in using anticonvulsants as needed. The INSET is all about is eliciting generally episodic symptoms.

Our experience over six years has shown this to be correct, and it is rare that we do not also administer its companion test, the INSET (Inventory of Neppe of Symptoms of Epilepsy and the Temporal Lobe) at a similar time. The INSET effectively screens episodic phenomena in the higher brain including seizures and particularly focuses on the temporal lobe because that is the location of many episodic or paroxysmal events. By contrast, the SOBIN allows us to elicit and then amplify static, more chronic, more maintained events. Effectively, the INSET measures more state phenomena, the SOBIN more trait phenomena. The INSET therefore screens for episodic or paroxysmal conditions, the SOBIN for chronic higher brain disabilities.

\section{The BROCAS SCAN: its common application with the SOBIN}

The practice of medicine is based on history taking, examinations, and confirmations, which may include investigations or further tests or clinical evaluations. In effect, the SOBIN is the trait of higher brain history taking, and the BROCAS SCAN, is the objective examination Evaluations comparing the objective examinations linked with the SOBIN:

Whereas higher brain function abnormalities, such as frontal lobe dysfunction, are very pertinent, these mainly involve physical signs found on examination. We can often pick up clues to frontal lobe disease on the SOBIN. However, we cannot pick up temporal lobe phenomena with overt physical signs very easily, unless there's a structural abnormality, such as a tumor. This is the purpose of the BROCAS SCAN (the BROCAS: Screening Cerebral Assessment of Neppe).

The BROCAS $\mathrm{SCAN}^{4,5}$ is another instrument developed by Vernon Neppe, this time in the early 1990s. It has been enormously successful in performing what it purports to do, namely higher brain examination at a consistent and excellent level. 'BROCAS' is an acronym for behavior, and then 10 significant higher 'ROCAS' brain categories namely, Recall, Recognition, Orientation, Organization, Concentration, Calculation, Apraxia, Agnosia, Speech and SensoryMotor-Reflex.
It is a testament to the BROCAS SCAN that over almost three decades it has never required changes, or changes in fundamental scoring, despite this being done by psychology students and graduates, not by physicians, and that, though no direct formal comparison has been done, it appears to elicit similar information to formal, structured neuropsychological testing, and results have been reliable and valid enough to be confirmed by someone ranked by his peers as a leading neuropsychiatrist ${ }^{1}$. But the availability of pertinent history-taking, as with the SOBIN has proven a great boon. Let's look at it in some detail because effectively we're discussing the SOBIN with the INSET and in this instance, the BROCAS SCAN, another test we commonly use.

\section{BROCAS and MOCA: which one should we use with the SOBIN?}

Based on nearly three decades of clinical experience, the BROCAS SCAN is, in our opinion, consistently far better than the Folstein MMSE (FMMSE) ${ }^{5}$ - in fact, at an entirely different order of magnitude in sensitivity, reliability, validity and specificity. A relatively new, fashionable and much more competitive assessment of mental status is the MOCA (Montreal Cognitive Assessment). ${ }^{6-12}$ The MOCA has become popular and well known. We have performed a MOCA-1 on all patients for about two years whenever we have done the BROCAS SCAN.

The BROCAS SCAN, like the MOCA, is an objective bedside assessment test to elicit physical signs of higher brain function. Neppe originally described it in 1989, and our non-medical staff, can perform this test with facility and accuracy generally in half-an-hour. The BROCAS is a unique, comprehensive detailed clinical standardized, validated, Bachelor's level, bedside screening, clinical evaluation. It applies 10 composites categories and in the SOBIN we have recently modeled on these. The BROCAS scan, is a test of 10 different higher cerebral cortical functions. A perfect score is zero. An individual with normal intelligence without major psychopathology generally scores a total of $<15$. The maximum score is 100 - very grossly impaired. Each of the 10 functions is scored 0-10 and Behavior is also assessed (MBRS). The BROCAS SCAN concentrates on physical signs and areas of the cerebral cortex such as the temporal lobe and limbic system, predominantly, symptom profiles. It is very sensitive, and broad spectrum not just evaluating concentration (as in another test, the IVA) or memory largely as in the Folstein MMSE. It is particularly useful to differentiate organic and functional illness, and annual follow-up allows measuring changes. It is routinely valuable initially with occasional follow-up in all neuropsychiatric patients. It is used with other clinically structured questionnaires including the SOBIN. It has regular clinical and forensic applications and is useful in malingering, neuropsychiatric diagnosis, and repetitive monitoring.

The BROCAS SCAN should ultimately lead to us being able to correlate objective brain signs in the BROCAS SCAN with the historical SOBIN.

We have collected data over 28 years on the BROCAS SCAN. Because we always compare it, we can say definitively that we've proven it to be profoundly better than the Folstein MMSE in every

\footnotetext{
${ }^{1}$ With respect, Dr. Vernon Neppe has been ranked under 'Neuropsychiatry' in every edition of the peer-reviewed America's Top Doctors, and prior to that Best Doctors in America. He also led the USA and International Delegation in the disciplines of Neuropsychiatry and Psychopharmacology. He happens to have reviewed and examined in detail every patient in this population of patients with BROCAS SCANs at the PNI.
} 
population group (normal brain function; mild cognitive impairments; dementia; focal disease).

\section{Balance}

Since the Montreal Cognitive Assessment (MOCA) has been added, we have also compared the BROCAS with the MOCA. The MOCA is certainly a quick reasonable measure but with limitations such as distortions in scoring in focal disease particularly. Moreover, some items that normal people may find difficult (for example, drawing cubes, and naming animals - cultural) diminish it's specificity to differentiate 'normal' from specific 'abnormal pathologies'. Consequently, the BROCAS is far more sensitive, a better screen and far more versatile across populations.

Based on our experience, the BROCAS SCAN appears to be much more thorough, significantly more sensitive, more reliable and more specific, yet more consistent than the MOCA. Yet, we have not formally published this data yet, and the difference is not an order of magnitude like with the FMMSE, but nevertheless, still profound. There are predictable limitations to the MOCA that the BROCAS SCAN does not have, and yet the converse does not appear to apply, other than time duration of the testing.

There's a necessary disadvantage to the BROCAS SCAN, too. This is so as appropriate administration of the BROCAS SCAN takes longer, about half-an-hour. This is possibly three-times longer than the MOCA, which takes about ten minutes, and six-times longer than the FMMSE, which takes about five minutes. However, information must be gleaned appropriately, diagnostically and logically and this is why the BROCAS SCAN is thorough, and for perspective, it is much better than several hours of neuropsychological testing.

\section{But let's just re-examine the MOCA. Perhaps a shorter test may be easier? The MOCA}

As an example of our choice of the BROCAS SCAN over the MOCA, we've found that the MOCA has limitations in scoring sensitivity (e.g. scoring just one for drawings), significant cultural limitations (e.g. the cube and animals), breadth of items, sensitivity even between categories, and at times making the test too easy (such as giving written instructions that assist in drawings). Moreover, the reliability of alternative versions of the MOCA, which have significant variations, is unacceptable. ${ }^{13}$ The MOCA population was designed to be limited to patients with mild cognitive impairment and that is certainly an important MOCA diagnostic subgroup. ${ }^{14,15}$ However, the MOCA is now being applied in some other conditions like stroke, ${ }^{7,8,10}$ Parkinson's disease ${ }^{16}$ and across cultures. ${ }^{17-19,15,17,20}$ Regrettably, we anticipate that the extended diagnostic clinical and cultural applications might exceed its potential value because of validity, reliability, sensitivity and specificity issues: Using single items covering multiple areas combined with the variation in test results and the need to label patients based on single scores might place patients variably and possibly inappropriately in different categories of impairment. Moreover, the necessary monitoring of repeat impairment has been shown to be unreliable or inconsistent: This is a major concern because change may be missed.

Nevertheless, the MOCA contains at least one item in the 10 major 'ROCAS' categories. That is an excellent advance over the very poor test that constitutes the Folstein MMSE: ${ }^{7,9,10,21}$ Our experience in our neuropsychiatric population, is that involving only one item in a category is inappropriate and misleading: It can easily imply that the MOCA is still purporting to measure functions, when that is at a questionable level of validity: ${ }^{15,17,20}$ The MOCA can easily underreport in a broader population, or inappropriately report pathology due to misinterpretations. To base opinions on only one specialized cognitive ability makes the valid interpretations much more clinically difficult. This is particularly so when the items that are used in the MOCA vary in difficulty across categories, and aggravated even more when it is inconsistently across versions. Variations in scoring of say $20 \%$ are worrisome across versions, particularly when cut-offs are so restricted ( 25 vs $26 ; 29$ vs $30 ; 20$ or less for example): The same applies to the FMMSE but much, much more so. ${ }^{7,9,10,21}$ By comparison, the BROCAS SCAN has several items that cover each of the ROCAS areas, and, additionally, it has an extra layer of validity in that the SOBIN, for example, applies at least 7 such screening questions to each ROCAS category: However, the SOBIN is a history-taking test not an objective evaluation, but one always done with an objective measure (the BROCAS SCAN).

Consequently, it is advisable to use the SOBIN with a MOCA examination, too. Moreover, the clinician would be using a questionnaire that he does not need to administer, just examine the pertinent results as he would do for history-taking. However, when choosing one over the other, we cannot at this point have a test that does not do what it purports to do. The MOCA is better than the FMMSE, but because of validity issues, cannot be seriously applied in evaluations at this point, no matter how much time we want to save.

\section{Cluster analyses and the SOBIN}

In this paper, we cluster together some of the features of the SOBIN. For example, is recognition of faces in so-called prosopagnosia linked up with recognition of flowers? What is the difference between the recognition of the face in prosopagnosia and the application of putting names onto faces? This is something highly pertinent because we are able to eventually develop population statistics.

Below are a few illustrations just to give a perspective, so you can see these actual questions in the abbreviated SOBIN that we are attaching. We illustrate the SOBIN questionnaire (a copyrighted instrument) with the first 20 questions out of what now, with the SOBIN-1 and the SOBIN-2, is in total 200 items. We're using the version here that we've used since its inception. As it happens, after writing this article, we reviewed the SOBIN structure in detail, and we're making the questions tighter, shorter, and hopefully, easier. However, these changes are not included here as we don't have data yet. We also have introduced 4 answer columns including one relating to symptoms post-injury and a qualitative differentiation of how the patient was post-injury compared to today.

\section{Please let us know about any possible injury to the brain and the date (Table I) (Table2).}

*We have listed only 20 of the 88 items in the SOBIN-1, as examples. We have not numbered in these 20 items anything from the recently implemented SOBIN-2 which has some 112 more additional items using the same ROCAS categories as in the SOBIN-1. This makes 200 items total. After the 88 -item SOBIN-1, we illustrate how we screen further by applying the accessory items (Table 3). There are also10 more items, A to $\mathrm{J}$ in the SOBIN-2 on possible causes. But they're now unified into one instrument the SOBIN so the differentiation is just historic and for statistical analyses and comparison of the items. 
Table I The SOBIN-I

Instructions: The following questions help us better understand your situation.

Some questions are complicated. Some are similar. Some may not appear relevant to you as the questionnaire is very broad:

So, if you have any difficulties, answer "?"

Please answer honestly: each question is important.

*Answers will be kept confidential.

You may choose not to answer a question; if so, please indicate that you do not wish to answer.

Check the relevant brackets $(\mathrm{x})$

a. Which hand do you write with? ( ) Right ( ) Left ( ) Either ( ) Uncertain

b. Which side do you bat or play tennis with? () Right () Left () Either () Uncertain

c. Which arm do you prefer to throw with? ( ) Right ( ) Left ( ) Either ( ) Uncertain

d. Which hand do you use scissors with? () Right ( ) Left ( ) Either ( ) Uncertain

e. Which side do you kick with? ( ) Right ( ) Left ( ) Either ( ) Uncertain

f. Which eye do you look down a microscope (try it if you need to!)? ( ) Right ( ) Left ( ) Either ( ) Uncertain

g. Which ear do you use on a phone? ( ) Right ( ) Left ( ) Either ( ) Uncertain

h. Which arm is stronger (dominant)? () Right ( ) Left ( ) Either ( ) Uncertain

i. Which position do you usually sleep on? () Right ( ) Left ( ) Either () Uncertain ( ) back ( ) tummy

Please re-look at those items in this section that you have answered "( $x$ ) either".

Have you actively trained yourself to use your less dominant side? ( ) No ( ) Yes: circle which item: a b c d e f g h i

The questions that follow ask about how well you can do certain things compared to most people you know. We want you to compare how you do things now, with times prior to any specific illness or brain injury you may have had or with an earlier time in your life. So, under the first column, $C=$ Current, please answer how you currently experience the task (for example, this month) and under the second column, $\mathrm{P}=$ Past, please answer how you were previously (for example, before an injury or before your current difficulties began; if you cannot identify a time when you began to have difficulties or if you have never had difficulties, when you were a teenager or young adult.

\title{
Scoring
}

If you have about the same difficulties as most other people, answer " $n$ "= normal, no real difficulty

If you experience difficulties from time to time, a slight amount or not too obviously, "s"= slight difficulty

If you frequently experience difficulties that are obvious to others or marked deviations from what most others seem to do or if most children can do the skil better than you, answer "g"= great difficulty

If you cannot decide between s (slight) and g (Great) choose s.

If you are uncertain whether you are normal or have any kind of difficulty, or if you cannot give a proper judgment of how you do in that task, or haven't noticed or haven't tested it, answer "u"= unsure or unknown

If you are far better than most people at the skill, or it is among your greatest strengths, "e"= exceptional

$P$ column $=$ past/prior to the injury

C column for currently i-

Table 2 First 20 items of the SOBIN-I

$$
\begin{aligned}
& \text { "n" = normal, no real difficulty } \\
& " \mathrm{~s} "=\text { slight difficulty } \\
& " \mathrm{~g} "=\text { great difficulty } \\
& \text { "u" = unsure } \\
& \text { "e" = exceptionally better at than most people } \\
& \text { C column for currently as compared to after injury } \\
& \text { P column = past/prior to the injury }
\end{aligned}
$$

\begin{abstract}
I Do you have difficulty keeping a rhythm?
\end{abstract}
2 Do you have difficulty singing a tune? 
Table Continued

$\begin{array}{ll}3 & \text { Do you have difficulty hearing tones i.e. are you "tone deaf"? } \\ 4 & \text { Do you have difficulty copying (mimicking, imitating) the voice or accent of others? } \\ 5 & \text { Do you have difficulty distinguishing left from right without thinking about it? } \\ 6 & \text { Do you have difficulty reading maps? } \\ 7 & \text { Do you have difficulty with street directions like North or South? } \\ 8 & \text { Do you have difficulty knowing the direction you're walking or finding your way back from a trail? } \\ 9 & \text { Do you have difficulty recognizing the faces of people you have met only once or twice? } \\ 10 & \text { Do you have difficulty putting names to faces that you recognize? } \\ 11 & \text { Do you have difficulty recognizing different movie characters? } \\ 12 & \text { Do you have difficulty recognizing flowers? } \\ 13 & \text { Do you have especial difficulty copying pictures? } \\ 14 & \text { Do you have especial difficulty drawing pictures? } \\ 15 & \text { Do you have difficulty putting together the pieces of game puzzles? } \\ 16 & \text { Do you have difficulty understanding what you are reading? } \\ 17 & \text { Do you have difficulty due to being a slow reader? } \\ 18 & \text { Do you have difficulty with finding simple words e.g."It's on the tip of my tongue"? } \\ 19 & \text { Do you have difficulty due to mixing up the order of digits/numbers e.g. phone numbers? } \\ 20 & \text { Do you have difficulty making simple calculations correctly? }\end{array}$

Table 3 Accessory items of the SOBIN

A) Are there any other skills you feel you have great difficulty with? Please list them:

B) Are there any other skills you feel you have great strength with? Please list them or circle from the following:

RHYTHM. TONE. IMITATING VOICES. SENSE OF DIRECTION. FACES. REMEMBERING NAMES. COPYING. DRAWING. CALCULATING. ROBLEM SOLVING. SPELLING. USING WORDS. PUZZLES. WORD LETTER GAMES.QUICK WITH FAMILIAR TASKS. WRITING NEATLY. FINE MOTOR TASKS. CREATIVE IDEAS. ARTISTIC. INTUITIVE. HUMOR.

C) How would you rank your overall intelligence compared with the general population?

( ) BM: Below average in most areas. ( ) BV: Varies a great deal but overall below average.

( ) AM: Average in most areas. ( ) AV: Varies a great deal but overall average.

( ) IM: Slightly above average in most areas. () IV: Varies greatly, overall slightly above average.

( ) VM: Very above average in most areas. ( ) VV: Varies greatly but overall very above average.

Please answer the following questions (even if lights don't bother you):

1. Using the worst scenario, what is your response to flashing lights? ( ) Really like them. ( ) Like them a little. () Neutral. ( ) Dislike them a little. ( ) Really dislike.

2. Using the worst scenario, check which lights trigger unpleasant symptoms?

() No lights problem.

()Flashing lights strobe. () Fluorescent lights. () Ordinary lights. () Moving lights from police or other vehicles. () EEG strobe lights. () Discothèque lights. () Some computer monitors. () When traveling through shadows of trees. Which are the worst of these?

3. Which lights sometimes cause symptoms even during a symptom free period?

() No lights problem. ()Flashing lights strobe. () Fluorescent lights. () Ordinary lights. () Moving lights from police or other vehicles. () EEG strobe lights. () Discothèque lights. 
Table Continued

() Some computer monitors. () When traveling through shadows of trees.

4. How frequently do such lights cause these problems when exposed to them?

() Never. () Rarely. () Monthly. () Weekly. () Daily. () Constantly. () When symptom-free.

5. Please check ALL symptoms you have had when lights have bothered you:

() No symptoms. () Migraine. () Other headache. () Seizures. () Blackouts spells.

() Pains outside head. () Vision problems. ()Hearing problems. () Nausea. () Vomiting.

() Psychic awareness. ()Visions. () Depression. () Agitation. () Mood fluctuations.

() Smells. () Tastes. () Sounds. () Skin sensations. () Other:

6. Please check the major symptoms you usually have when lights have bothered you:

() No symptoms. () Migraine. () Other headache. () Seizures. () Blackouts spells.

() Pains outside head. () Vision problems. ()Hearing problems. () Nausea. () Vomiting.

() Psychic awareness. ()Visions. () Depression. () Agitation. () Mood fluctuations.

() Smells. () Tastes. () Sounds. () Skin sensations. () Other:

These questions are about other perceptions that may trigger your symptoms:

1. Do you ever have symptoms such as the above triggered by any other perceptions?

() No. Yes: ()Sounds. () Smells. () Tastes. () Skin sensations. () Balance disturbances.

() Menstrual cycle. () Other:

2. How often?

() Never. () Rarely. () Monthly. () Weekly. () Daily. () Constantly. () When symptom-free.

3. Which symptoms?

() No symptoms. () Migraine. () Other headache. () Seizures. () Blackouts spells.

() Pains outside head. () Vision problems. ()Hearing problems. () Nausea. () Vomiting.

() Psychic awareness. ()Visions. () Depression. () Agitation. () Mood fluctuations.

() Smells. () Tastes. () Sounds. () Skin sensations. () Other:

Would you say all your answers to the questions above describe accurate information about you?

( ) Yes, I’ve tried my best. ( ) Mainly so. ( ) Marginally. ( ) No, this is not accurate.

\section{Broadly, possible causal factors (new questions introduced in the SOBIN-2)}

Do you have difficulty due to a problem during your own birth e.g. limited oxygen to your brain?

Do you have difficulty due to a brain injury with some lack of consciousness (even just for a few seconds)?

Do you have difficulty due to a concussion?

Do you have difficulty due to not remembering a traumatic event?

Do you have difficulty due to seizures?

Do you have difficulty due to a coma?

Do you have difficulty due to a skull fracture?

Do you have difficulty due to meningitis?

Do you have difficulty due to a tumor?

Do you have difficulty due to a medical disease? If so, what? 


\section{Clusters by function}

The SOBIN is a copyrighted instrument that is valuable in clinical use and if appropriate, may be billed in testing. It is available to others as a research instrument. We invite collaborations in this regard.

For example, we have recently classified the SOBIN by its items, clustering them together. Cluster analysis can allow for demonstrating internal validity. It can also imply which items are redundant.

We list the items from the SOBIN clustered together. The additional new (SOBIN-2) items are not numbered below. The different items in the SOBIN history-taking instrument have been summarized under the categories we apply the same item heads as in the BROCAS SCAN examination. These are convenient for research analyses but also logical as possibly the best available way to cluster the items. The BROCAS items are listed below.

\section{Behavior}

Do you have difficulty with "hyperactivity"? i.e. do you have difficulty keeping still, for example, restlessness with pacing, drumming of fingers, shifting position while sitting, leaving a table or room frequently, feeling edgy while at rest?

Do you have a poor sense of humor or is it exceptional?

Do you have difficulty due to becoming easily frustrated, giving up quickly, and/or lacking self-confidence?

\section{The BROCAS uses the Revised Brief Psychiatric Rating Scale}

This test uses a 0-6 (occasionally 7) ordinal ranking scale on 18 basic items; 3 COP (concentration, orientation, and perplexity) items and an additional frustration score and in addition to orientation. The 18 basic items are psychopathology items, derived from the MBPRS. A "validity score" is based on whether particular items could be ranked accurately (0), questionably (1), or not at all (2).

On the SOBIN, the emphasis is on subtle symptoms, not psychopathology or behaviors.

Some of the items overlap and can be classified elsewhere on the ROCAS, fulfilling more than one purpose.

We number the items from the SOBIN-1, and differentiate them from the SOBIN-2 which is not numbered.

\section{R: Recall-memory}

2 items S1, 5 items $\mathrm{S} 2=7 \mathrm{~S} .(\mathrm{S} 1=$ Sobin- $1 ; \mathrm{S} 2=$ Sobin 2$)$

When interrupted, do you tend to lose the theme of simple tasks you were doing?

When going from one room to another in your house, do you find you forget what you intend to do?

Do you have difficulty remembering information when playing games?

Do you have difficulty due to being forgetful?

Do you have difficulty due to forgetting to pay bills or other common tasks of great importance?

Do you get lost while driving?
Do you get confused: You don't know where you are, or what happened?

\section{R: Recognition—memory}

0 items $\mathrm{S} 1,7$ items $\mathrm{S} 2=7 \mathrm{~S}$

Do you need to be prompted in order to remember things?

After you were interrupted, do you have difficulty remembering what you thinking or about to say before?

Do you have difficulty recalling things you were asked to remember without clues?

Do you have difficulty remembering things due to being easily distracted?

Do you have difficulty remembering to do something even after you were given a reminder?

Do you have difficulty taking care of things on a list even when given reminders?

Do you have difficulty remembering everything? "Memory like a sieve - forget everything."

\section{O: Orientation}

3 Items S1, 6 items S2.= 9S

Orientation involves for time, space including directional, and passage of time. Passage of time is selectively impaired in certain psychoses; spatial impairment may imply organicity.

\section{Directional orientation}

Do you have difficulty reading maps?

Do you have difficulty with street directions like North or South?

Do you have difficulty knowing the direction you're walking or finding your way back from a trail?

Do you have difficulty keeping track of what the date is?

Do you have difficulty keeping track of what day of the week it is?

Do you have difficulty keeping track of what month it is?

Do you have difficulty keeping track of what year it is?

Do you have difficulty keeping track of what place you're in?

Do you have difficulty figuring out the way to go? (e.g., do you have difficulty pointing to the direction you would go to get home?)

\section{O: Organization}

15 items S1, 5 items $\mathrm{S} 2=20 \mathrm{~S}$.

Organization as reflected by abstraction of proverbs and ability to coherently put elements together appear within normal limits taking cultural elements into account.

The measure in this instance, unfortunately, has more cultural elements than any other part of this test.

\section{Multitasking}

Do you have difficulty doing two simple tasks at once (for example, playing a game, and also talking)? 


\section{Reading}

Do you have difficulty understanding what you are reading?

Are you a slow reader?

\section{Dysproccia}

\section{S1. 6. 52 4. $S 10$}

Do you take a long time to complete tests?

Do you find that projects or homework take you a long time to do?

Do you find that you do tasks slowly?

Are you slow with most tasks?

If yes, is this only because you feel you must carefully check everything? () yes () no

Are you clumsy i.e. do you find that unless you are especially careful, you are more likely to break or spill things, or mess on your clothes more?

Do you have difficulty with learning?

Do you have difficulty completing simple projects in a typical amount of time? (e.g. Does a 30-minute task take you hours?)

Do you have difficulty due to taking much longer to fill in forms?

Do you have difficulty focusing your attention?

Do you need extra time at school to complete tests?

\section{Executive functions}

Do you have difficulty putting together the pieces of game puzzles?

Do you have difficulty typing on a keyboard despite much typing experience?

Do you have especial difficulty copying pictures?

Do you have difficulty with copying shapes and drawings?

Do you have difficulty copying a sequence of movements you've been shown?

Do you regard yourself as having difficulty with attention?

Do you have difficulty mixing up "receivables" or "payables" in banking?

\section{C: Concentration}

\section{Concentration and attention}

5 items $\mathrm{S} 1,3$ items $\mathrm{S} 2=8 \mathrm{~S}$

Concentration impairments are very non-specific.

Do you have difficulty concentrating for long periods of time, even when you are interested (for example in a teaching or learning situation when you are not tired)?

Do you lose your train of thought (e.g. you're talking about something then forget what)?

Do you find that you forget what you want to say when others are talking first?

Are you easily distracted?
In the presence of noise, do you have difficulty with simple tasks while doing work or study?

Do you have difficulty doing more than one simple task at a time?

Do you have difficulty doing more than one complicated task at a time?

Do you have difficulty paying attention for an entire episode of a TV show that you like watching?

\section{C: Calculation}

6 Items S1, 6 items S2 $=12 \mathrm{~S}$

\section{Calculation}

Calculation skills in the BROCAS are measured by subtraction and difficulties may reflect specific impairments including preexisting learning difficulties and focal impairments of either parietal or frontal lobe or generalized concentration disturbance. The SOBIN moves beyond the BROCAS.

Do you have difficulty getting simple calculations correct?

Do you find you can calculate well, but only with a pen and paper, not easily in your head?

Do you find that you can calculate well but it takes you a long time to get the answer?

Do you have difficulty solving math problems or stories or riddles - for example, at school could you do the simple arithmetical calculations but found that when translated to stories or concepts or problems, you had difficulty?

Do you have difficulty with multiplication tables under 10 (e.g. 5 times 6)?

Do you have difficulty with multiplication tables above 10 (e.g. 11 times 12)?

Do you have difficulty doing simple calculations like subtraction in your head?

Do you have difficulty doing simple calculations like addition in your head?

Do you have difficulty doing simple calculations like division in your head?

Do you have difficulty doing simple calculations like multiplication in your head?

Do you have difficulty doing very simple calculations like addition together with subtraction in your head? (e.g. 4+8-3)

Do you have difficulty because your calculation skills have changed? (e.g. become worse or better?)

\section{A: Apraxia, motor-praxis}

Praxic skills pertaining, inter alia, to copying, construction, and sequential movements. It is difficult to ask questions in the SOBIN about sequential movements so here the BROCAS and SOBIN work together again.

\section{Executive praxis}

7 Items S1, 1 items S2.= 8S 
Do you have especial difficulty drawing pictures?

Would you regard yourself as having problems with painting artistically?

Would you regard yourself as having problems with creative ideas (it is difficult to have problems here because most people do not exhibit creative ideas; but maybe you're exceptional at it)?

Do you have difficulty tying a knot?

Do you have difficulty tying shoelaces?

Do you have difficulty tying ties?

Do you have difficulty pushing and pulling doors?

Do you have difficulty drawing clocks?

\section{A: Agnosia, sensory, gnosis}

Gnosic elements test perceptual modality (auditory, visual, tactile) and organization of information. The SOBIN extends this particularly with prosopagnosia and higher perceptual functions. The BROCAS. test involves relatively simple tasks so abnormality reflects significant pathology or limited attention to detail. The SOBIN again amplifies.

25 items S1, 3 items $\mathrm{S} 2=28 \mathrm{~S}$

\section{Prosopagnosia}

6 items S1, 0 items $\mathrm{S} 2=6 \mathrm{~S}$ )

Do you have difficulty recognizing the faces of people you have met only once or twice?

Do you have difficulty putting names to faces that you recognize?

Do you have difficulty recognizing faces of actors in movies/plays when scenes change?

Do you have difficulty recognizing different movie characters?

Do you have difficulty recognizing pictures or statues?

Do you have difficulty recognizing flowers?

\section{Perceptual auditory recognition}

Do you have difficulty recognizing voices on the phone of people you know well?

Perceptual visual recognition

Do you have difficulty recognizing colors??

Do you have difficulty with recognizing shapes and drawings?

Do you have difficulty with recognizing places you have visited infrequently?

Do you have difficulty doing Jigsaw puzzles?

\section{Perceptual visualization}

Do you have difficulty telling time with watches that have hands (not digital numbers)?

Do you have difficulty telling time with watches that have numbers (not hands)? front?

Do you have difficulty with positions of objects like back from

\section{Gnosis}

Do you have difficulty distinguishing left from right without thinking about it?

Do you have difficulty distinguishing which of your fingers is which?

Do you have difficulty recognizing one side of your body as yours?

Do you have difficulty touching your toes while keeping your legs straight?

\section{Auditory Prosody}

Do you have difficulty singing a tune?

Do you have particular difficulty hearing tones i.e. are you "tone deaf'?

Do you have difficulty keeping in tune?

Would you regard yourself as having problems with your sense of humor?

Do you have difficulty humming a melody without words?

Do you have difficulty humming your own tune without words (e.g. da-da-do-da-da-do)

\section{Higher perceptual functions}

Visual

Do you see double sometimes (even though your glasses are adjusted)?

Are you color blind? If so, to what degree?

Do you have difficulty with information that flashes onto screens or posters?

\section{Gustatory}

Do you have difficulty tasting foods (when you don't have a cold or such condition)?

\section{Olfactory}

Do you have difficulty smelling things (when you don't have a cold or such condition)?

\section{S- Speech}

Speech pronunciation, Communication skills, Comprehension of complex English phrases, Naming of body parts, colors, and objects as well as Speech generation as reflected by spontaneous word generation using stipulated criteria are all tested in the BROCAS which broadly cover the spectrum of receptive and expressive speech. The SOBIN extends beyond this but cannot test some BROCAS screen items. Again, the two work together.

12 items S1, 0 items S2 = 12S (4 categories)

\section{Language, speaking. Executive}

Do you have particular difficulty copying (mimicking, imitating) the voice or accent of others?

Naming. 
Do you have difficulty with finding simple words (i.e. "It's on the tip of my tongue")?

Do you have difficulty thinking of the correct word even though the word is relatively simple?

Do you have difficulty with putting a name to simple things you are shown?

Do you have difficulty naming colors?

\section{Receptive speech}

Do you have difficulty speaking even though you know the exact words you want to say?

Do you have difficulty understanding certain sentences, even when they are at a level that you would expect to understand?

\section{Speech ordering}

Do you frequently mix the order of digits (numbers) for example in a telephone number?

Do you have difficulty putting together scrambled letters - for example, in games like Scrabble, in crossword puzzles or in anagrams?

\section{Writing}

Do you have difficulty spelling simple words?

Do you have difficulty using punctuation marks?

Do you have problems with writing neatly?

Do you have difficulty writing handwriting legibly so others can easily read it?

\section{Sensory-Motor-Reflex Elements}

In the BROCAS this reflected by gait, posturing, and muscle tone/ power plus obvious motor weakness in the upper limbs with no arm sway and no flexor-extensor weakness and the lower limb strength appears adequate. Motor tone appears adequate. No tremor occurs on either side either at rest or on writing. BROCAS assesses obvious sensory loss and primitive reflexes. Because broad elements only are tested this is no substitute for a detailed neurologic examination. In the SOBIN we cannot elicit a history of primitive reflexes and test for sensory loss.

\section{Sensory}

2 items $\mathrm{S} 1,5$ items $\mathrm{S} 2=5 \mathrm{~S}$ (2 categories)

Do you get dizzy?

Do you see double with just one eye open?

Do you have difficulty due to motion sickness in vehicles or seasickness on boats?

Do you have prickly sensations in your arms or legs for no reason?

Do you have ringing or buzzing in your ears?

If so, is it most of the time in your ( ) right, ( ) left or ( ) both ears?

\section{Rhythm}

1. Do you have difficulty keeping a rhythm?
Do you have difficulty with your ability to keep in step, for example when marching?

\section{Motor}

6 items S1, 55 items S2 = 28S (4 categories)

\section{Motoric (also partly a praxic function)}

Do you have difficulty with kicking balls?

Do you have difficulty with catching balls?

Do you have difficulty with hitting balls?

Do you have difficulty throwing balls accurately?

Do you have difficulty throwing balls or other objects straight with your main arm?

Do you have difficulty throwing balls or other objects straight with your other arm?

Do you have difficulty timing when to go for the ball?

Do you have difficulty using the appropriate pressure needed to putt a golf ball into the nearby hole?

Do you have difficulty using the appropriate pressure needed to type on a smartphone e.g. iPhone?

Do you have difficulty using the appropriate pressure needed to turn the page of a book?

Do you have difficulty using the appropriate pressure needed to hold and write with a pencil?

Do you have difficulty using the appropriate pressure needed to hold a cup of water?

Do you have weakness in one or both of your arms? body?

Do you have difficulty due to weakness in parts or one part of your

Do you have weakness on one side of your body?

Do you have weakness in a particular area of your body?

Do you have difficulty due to tremoring/shakiness e.g. in one or both of your hands?

Do you have difficulty due to your legs moving/shaking?

Do you have difficulty due to repetitive movements while sitting e.g. constantly tapping your foot?

Do you have difficulty due to your body moving slowly?

Do you have difficulty due to your joints being stiff?

Do you have difficulty due to your muscles being stiff/rigid?

Do you have difficulty due to your muscles being fatigued easily?

Do you have difficulty due to your body being unable to do what your brain is telling it to do?

Do you have difficulty using a toothbrush e.g. cleaning all the necessary places in your mouth?

Do you have difficulty figuring out how you need to move your body when getting dressed? 
Do you have difficulty figuring out how you need to move your body when getting undressed?

Do you have difficulty due to being uncoordinated e.g. tripping over your own feet?

Do you have difficulty due often tripping and actually or very nearly falling?

Do you have difficulty due to frequently bumping into things or people accidentally?

Do you have difficulty due to being unaware of a part or parts of your body?

Do you have difficulty with keeping in time while dancing?

Do you have difficulty walking forwards with your eyes closed?

Do you have difficulty walking backwards with your eyes closed?

Do you have difficulty walking heel-to-toe e.g. when you take a step, does your heel comes down to the ground before the ball of your foot and your toes?

Do you have difficulty climbing?

Do you have difficulty running?

Do you have difficulty riding a bicycle?

Do you have difficulty doing jumping jacks?

Do you have difficulty skating e.g. roller-skating/blading or iceskating?

Do you have difficulty swimming?

Do you have difficulty going up stairs?

Do you have difficulty going down stairs?

Do you have difficulty with stairs due to being frightened by them?

Do you have difficulty going up escalators?

Do you have difficulty going down escalators?

Do you have difficulty with escalators due to being frightened by them?

Do you have difficulty using moving walkways (e.g. at airports)? Is it because you are afraid of them? ( ) yes or ( ) no

Do you have difficulty using elevators? Is it because you are afraid of them? ( ) yes or ( ) no time?

Do you have difficulty due to appearing limp and lethargic all the

Do you have difficulty due to needing to rest your head on your hands or lay your head down e.g. on your arms at a desk/table?

Do you have difficulty due to slumping e.g. at your desk, at the dinner table, etc.

Do you have difficulty maintaining the necessary posture for certain activities? If so, what?

Do you have difficulty finding and maintaining a comfortable seated-posture?
Do you have difficulty due to typing too slow? If you don't type, put in ' $u$ ' for unknown.

Do you have difficulty typing accurately?At what speed?

Do you have difficulty due to feeling unsafe doing certain kinds of movement?

Do you have difficulty due to poor balance?

Do you have difficulty standing on one foot?

Do you have difficulty with tasks that require balancing? If so, what?

Do you have difficulty due to walking too hard or too loudly?

Do you have difficulty due to pushing things too hard?

Do you have difficulty due to banging things too hard or too loudly?

Do you have difficulty due to writing too hard?

Do you have difficulty due to playing with things too hard or too roughly?

Do you have difficulty due to being "the loud one"?

Do you have difficulty due to being "the rough one"?

Do you have difficulty due to crashing/banging into things?

Do you have difficulty due to moving/shaking/running/jumping/ bouncing around too much like an insatiable bundle of energy?

Do you have difficulty due to hurting yourself?

Do you have difficulty due to hurting others?

Do you have difficulty due to having to have certain clothes e.g. turtlenecks, tight belts, hoods, hats, jackets zipped all the way up, tight pajamas, etc.?

Do you have difficulty recognizing voices? (when you cannot see the person, e.g. on the phone even the line is clear).

Do you have difficulty pinpointing approximate times in the past? (You remember the event but cannot work out even a little how long ago it was)

\section{Reflex}

0 items S1, 2 items $\mathrm{S} 2=28 \mathrm{~S}$ (4 categories)

Do you have difficulty playing quick reflex ball sports e.g. tabletennis/ping-pong, squash, racquet ball?

Do you have difficulty playing quick reflex video games?

\section{Additional items in the new SOBIN-2}

In preparation for this paper, we again researched the literature and clinically examined out items.

In addition to the already available 88 items, we have added 112 more into what we're calling the SOBIN-2. We added these items because some areas were not adequate as we had them. There was insufficient memory recognition for example, and emphasis on proprioception. 
We briefly discuss two conditions: Prosopagnosia and Dysproccia

\section{Research}

The SOBIN provides a fertile way to obtain information on particular kinds of symptoms. In one instance, it might relate to, "how do certain symptoms correlate?"

\section{Prosopagnosia}

Take for example, the symptom prosopagnosia. Usually, we regard this as difficulty with facial recognition. On the Internet, the implication is that this is a rare phenomenon, maybe due to a rare tumor in the appropriate brain area. In fact, this is not rare; in our experience it may be one of the most common difficulties we encounter. This difficulty recognizing the faces of people whom one has just met once or twice is common.

There are two issues to resolve:

1. Does facial recognition difficulty correlate with putting names to the faces of people you recognize? How often do these occur together?

2. How does this correlate with poor recognition of an actor in a movie or in a play? Is this worse when scenes change or if the person is dressed differently? How much difficulty is there in recognizing different movie characters? Does this correlate with pictures, or statues, or recognizing flowers?

These then link with six different SOBIN items relating to prosopagnosia. They reflect how we can apply the SOBIN in research in cluster analyses: We examine whether or not these kinds of symptoms are related - and we think they are related except for putting a name to a face which may be entirely different.

Prosopagnosia has been postulated to be located in the fusiform gyrus. But can we prove it? Maybe: Sometimes there are exceptional skills in terms of recognition of patterns: For example, Susan Polgar, former women's world chess champion and grand master, was tested with PET. Her fusiform gyrus lit up greatly, while she was remembering not only positions in chess games but recognizing the actual pieces even when a bus that had a picture of the position was passing. 22 So, this linkup may be an important one.

\section{Dysproccia}

Let's take a second example, dysproccia. Neppe described the entity of what he called 'dysproccia' in 2001. We regard it as an extremely important symptom and an extremely common one, yet neglected.

The public classically talk about dyslexia and difficulty with calculation (as opposed to reading) or with writing. Often, they correlate this with Attention Deficit Disorder but recognize this as a cerebral cortical difference. However, it is far more practical recognizing how long it takes to process one's homework or one's work at work and one's performance in this regard. That is very important and very relevant. Questions such as, how difficult is it to do new tasks? Can one understand them? Or is one just slowed? Or translating neurologically: Is it the processing of the information as the input comes in? Or is it the output? These are key features pertaining to dysproccia, ${ }^{23}$ and this is another kind of symptom that one would try to evaluate.
These are higher perceptual functions and they might involve perceptual function (central sensory input) or they may be central in the brain (mixed) or reflect higher executive functions.

The cluster of tests pertains to how long it takes to complete tests: Do you find projects or homework take you a long time to do? Do you find that you do the tasks, but slowly? Are you slow with most tasks?

Thereafter, the evaluator must examine the detail. For example, an obsessional component may exist and is that primary or secondary to the disability? We can again cluster it: Does this correlate with clumsiness? Are you clumsier than others? And we can apply other examples: Do you find that unless you are especially careful, you are likely to break things or spill things or ruin your clothes easily? Do you have difficulty with learning?

So here is an example of how six different functions again are linked up with a particular higher brain function: dysproccia: They attempt to do a 30-minute homework assignment, but they end up taking hours, and they may drop out of school despite seeming intelligent.

Dr. Neppe described dysproccia some 17 years ago ${ }^{23}$, yet it doesn't even appear on the Internet. This was described in 2001 as 'dysproccia, a necessary new term for impairment of higher brain function. ${ }^{23}$ Neuropsychiatrists, neuropsychologists, child psychologists, educational psychologists, and teachers frequently see this commonly unlabeled educational disability, and this is one example of what the SOBIN can do. It can enhance diagnosis and differentiate this brainrelated difficulty in processing information from overt dyslexia, or dysgraphia, or dyscalculia, or anosognosia, or aprosodia, or executive apraxia, or agnosic difficulties in terms of knowing things, or aphasic or attentional deficits. They may exhibit none of these, and yet they cannot perform well.

Could it be that these patients are hardwired in a different way and they are regarded as slow or unintelligent, but when given time to perform tasks, they will do them successfully? They often need extra time at work. Yet we lacked a descriptive vocabulary, which is why Dr. Neppe introduced this term 'dysproccia' for people who have 'dysproccic' difficulties. Labeled some 15 years ago, and it has several different higher processing mechanisms-auditory, visual-motor, reading processing, or pure motor-which could ultimately relate to work disability. And these people need extra time and attention, but it can impair their functioning a great deal. And so it's of great practical and theoretical importance.

It is astonishing that this term, dysproccia, has not taken over the literature, because it is extremely important in terms of a specific kind of focal disability. One could also screen and see whether or not their birth was normal or whether there was any other brain damage. This is why cluster analyses of similar symptoms are important as one SOBIN function.

From an evaluation point of view, this is where conventional neuropsychology can very often fail. Tests don't assess what could be done in a brief period. But they do sometimes. They'll come along and say: "Oh, the patient is doing fine! We couldn't find any impairment here, or this or that or the other." The major thing is, if people take 20 minutes to do what should be taking 2 minutes, this is problematic. 


\section{Rehabilitation}

Part of the rehabilitation is picking up the strengths and the weaknesses, and specific focal deficits, such as prosopagnosia and dysproccia, are particularly missed.

\section{Conclusions}

There are several different conditions, which vary enormously in the so-called normal population and in the abnormal population. These can be immobilizing - sometimes even when they are single symptoms. Alternatively, patients can compensate totally and they or others might not even notice them. Everyone likely has some difficulty of some kind, and they adapt to this.

The SOBIN has proven very valuable and is the main focus of this article. However, it must be perceived as one of three major test instruments - two historical screens (INSET and SOBIN) and one evaluation of higher brain function (BROCAS SCAN) that are performed in the same patient as part of a three-fold examination. This has allowed us the PNI to evaluate in detail neuropsychiatric and behavioral neurological patients. However, the data even on psychiatry has been very adequate and lends itself to further collaborations. Moreover, therapeutically, the awareness of subtle higher brain dysfunctions allows the neuropsychiatrists, neurologists, psychiatrists, psychologists and other mental health professionals an insight into the patient's strengths and weaknesses of their higher brain function. This translates into better quality management and, if needed, rehabilitation. The computer era has made such approaches easier and more practicable than before.

We perceive the SOBIN as having a valuable future clinically, forensically, and in research. But we do not see it as adequate for use on its own at this point. The revision that we have done this month on the wording of the SOBIN items and clarification of definitions should increase its usability. Moreover, the more direct translation of the clusters of related symptoms should improve usability.

We can classify the higher brain subtle symptoms of the SOBIN by using the classic 10-point ROCAS scale of the BROCAS SCAN: recall, recognition, orientation, organization, concentration, calculation, apraxia, agnosia, speech, and sensory-motor reflex phenomena. This creates a valuable fabric for understanding and comparing symptoms.

In each instance, the SOBIN screens not for one symptom in each ROCAS category, as happens with even some objective tests such as the MOCA and (even less so with the Folstein mini-mental status). The SOBIN screens in detail. There are no right or wrong answers; although if there is a gross abnormality, one needs to truly compensate for more than the basic abnormalities.

Our application of two very neglected conditions - prosopagnosia and dysproccia-reflect examples of how to apply clinical data and ultimately research using the SOBIN.

\section{Acknowledgements}

Thank you to Biagio Longano, Dr. Judith Milner, Suzan Wilson, and to Jacqui Slade for her careful evaluations.

\section{Conflict of interest}

Authors declare there is no conflict of interest in publishing the article.

\section{References}

1. Neppe VM. Clinical and forensic applications of the SOBIN (Subtle organic brain inventory of Neppe) with the INSET (Inventory of Neppe of symptoms of epilepsy and the temporal lobe). J Neuropsychiatry and Clinical Neurosciences.

2. Neppe VM. Utility, applications, validity and reliability of the Inventory of Neppe of Symptoms of Epilepsy and the Temporal Lobe (INSET) compared with ambulatory electroencephalographic parameters, longitudinal clinical features, anticonvulsant responsiveness, and the SOBIN (Subtle Organic Brain Inventory of Neppe). American Epilepsy Society. Seattle, WA, American Epilepsy Society.

3. Neppe VM. Taming the temporal and frontal lobes of the brain by applying higher brain function structured inventories - "The Inventory Of Neppe Of Symptoms Of Epilepsy And The Temporal Lobe" (INSET) and the "Subtle Organic Brain Inventory Of Neppe" (SOBIN) together with ambulatory electroencephalography parameters, and clinical anticonvulsant responsiveness. J Neuropsychiatry and Clinical Neurosciences. 2015; $181-\mathrm{e} 182$.

4. Neppe VM. Diagnostic and follow-Up clinical utility of the BROCAS SCAN - The Screening Cerebral Assessment Of Neppe and Auditory and Visual Integration Test (IVA) in Neuropsychiatry, psychiatry and behavioral neurology outpatient clinical and forensic use. $J$ Neuropsychiatry and Clinical Neurosciences. 2015

5. Neppe V, Chen A, Davis et al. The application of the screening cerebral assessment of Neppe (BROCAS SCAN) to a neuropsychiatric population. J Neuropsychiatry Clin Neurosci. 192;4(1):85-94.

6. Gagnon JF, Postuma, RB, Joncas, S, et al. The Montreal Cognitive Assessment: a screening tool for mild cognitive impairment in REM sleep behavior disorder. Mov Disord. 2010;25(7):936-40.

7. Pendlebury ST, Cuthbertson FC, Welch SJ, et al. Underestimation of cognitive impairment by Mini-Mental State Examination versus the Montreal Cognitive Assessment in patients with transient ischemic attack and stroke: a population-based study. Stroke. 2010; 41(6):1290-1293.

8. Cumming TB, Bernhardt J, Linden T. The montreal cognitive assessment: short cognitive evaluation in a large stroke trial. Stroke. 42(9): 2011.

9. Damian AM, Jacobson SA, Hentz JG, et al. The Montreal Cognitive Assessment and the mini-mental state examination as screening instruments for cognitive impairment: item analyses and threshold scores. Dement Geriatr Cogn Disord. 2011;31(2):126-131.

10. Godefroy O, Fickl A, Roussel M, et al. Is the Montreal Cognitive Assessment superior to the Mini-Mental State Examination to detect poststroke cognitive impairment? A study with neuropsychological evaluation. Stroke. 2011;42(6):712-1716.

11. McLennan SN, Mathias JL, Brennan LC, et al. Validity of the montreal cognitive assessment (MoCA) as a screening test for mild cognitive impairment (MCI) in a cardiovascular population. J Geriatr Psychiatry Neurol. 2011;24(1):133-38.

12. Olson R, Tyldesley S, Carolan H, et al. Prospective comparison of the prognostic utility of the Mini Mental State Examination and the Montreal Cognitive Assessment in patients with brain metastases. Support Care Cancer. 2011;19(11):1849-55.

13. Price CC, Cunningham H, Coronado N, et al. Clock drawing in the Montreal Cognitive Assessment: recommendations for dementia assessment. Dement Geriatr Cogn Disord. 2011;31(3):179-187.

14. Nasreddine ZS, Phillips NA, Bedirian V, et al. The Montreal Cognitive Assessment, MoCA: a brief screening tool for mild cognitive impairment. J Am Geriatr Soc. 53(4):695-699. 
15. Lebedeva E, Huang M, Koski L. Comparison of alternate and original Items on the Montreal Cognitive Assessment. Canadian Geraitrics Journal. 2016;19(1):15-18.

16. Gill DJ, Freshman A, Blender JA, et al. The Montreal cognitive assessment as a screening tool for cognitive impairment in Parkinson's disease. Mov Disord. 23(7):1043-1046.

17. Lee JY, Dong Woo L, Cho SJ, et al. Brief screening for mild cognitive impairment in elderly outpatient clinic: validation of the Korean version of the Montreal Cognitive Assessment. $J$ Geriatr Psychiatry Neurol. 2008;21(2):104-110.

18. Wong A, Xiong YY, Kwan PW, et al. The validity, reliability and clinical utility of the Hong Kong Montreal Cognitive Assessment (HK-MoCA) in patients with cerebral small vessel disease. Dement Geriatr Cogn Disord. 2009;28(1):81-87.
19. You JS, Chen RZ, Zhang FM, et al. The chinese (cantonese) montreal cognitive assessment in patients with subcortical ischemic vascular dementia. Dement Geriatr Cogn Dis Extra. 2011;1(1):276-282.

20. Fujiwara Y, Suzuki H, Yasunaga M, et al. Brief screening tool for mild cognitive impairment in older Japanese: validation of the Japanese version of the Montreal Cognitive Assessment. Geriatr Gerontol Int. 2010;10(3):225-232.

21. Athilingam P, King KB, Burgin SW, et al. Montreal Cognitive Assessment and Mini-Mental Status Examination compared as cognitive screening tools in heart failure. Heart Lung. 2011;40(6):521-529.

22. Polgar S. My brilliant brain, in My brilliant brain. 2016.

23. Neppe VM. Dysproccia: a necessary new term for impaired higher brain processing. J Neuropsychiatry Clin Neurosci. 2001;13(3):428-429. 